\title{
Comportamiento Fenológico en tres rangos altitudinales de las especies Enterolobium cyclocarpum, Maclura tinctoria. Esteli, Nicaragua
}

\author{
Mariela Lisbeth Hernández Olivas ${ }^{1}$ \\ Beneyda Leticia Montalván Calderón ${ }^{2}$ \\ Ana María Flores Briones ${ }^{3}$ \\ Josué Tomas Urrutia Rodríguez ${ }^{4}$ \\ Kenny López Benavides 5
}

\section{RESUMEN}

El deterioro que presentan las áreas boscosas tropicales de Nicaragua como consecuencia de acciones antrópicas, ha traído como resultado la fragmentación y reducción de bosques. Actualmente los estudios fenológicos han tomado gran importancia, enfatizándose principalmente en procesos biológicos de supervivencia y éxito reproductivo de las especies, a fin de mejorar la distribución futura y adaptación a los cambios del clima; ya que se han observado variaciones en sus etapas fenológicas.

El estudio consistió en evaluar el comportamiento fenológico en tres rangos altitudinales de las especies arbóreas Enterolobium cyclocarpum, Maclura tinctoria del bosque tropical seco del Norte de Esteli - Nicaragua, con el propósito de obtener información sobre la fenología de estas especies, para contribuir a su recuperación y conservación. Se evaluó el comportamiento fenológico en tres rangos altitudinales: parte baja, parte media y parte alta. La observación de las características fenológicas se basó en la metodología de Fournier (1974). Los datos obtenidos se analizaron utilizando la prueba de varianza no paramétrica de Kruskal-Wallis. Los individuos de la especie M. tinctoria mostraron diferencia significativa en el porcentaje de hojas, rebrotes y frutos en el rango altitudinal de 700 a $800 \mathrm{msnm}$; y E. cyclocarpum fue solamente en hojas y rebrotes en el rango altitudinal de 900 a $1000 \mathrm{msnm}$. La especie Enterolobium cyclocarpum es la que mejor se adapta a diferentes alturas, comportándose de manera similar, recomendada para utilizarla en programas de reforestación.

Palabras claves: Fenología, rangos altitudinales, Maclura tinctoria, Enterolobium cyclocarpum.

1 UNAN-Managua/FAREM-Estelí. Correo electrónico: marielalisbethhernandez@yahoo.es

2 UNAN-Managua/FAREM-Estelí. Correo electrónico: mbeneydaleticia@yahoo.com

3 UNAN-Managua/FAREM-Estelí. Correo electrónico: afloresbriones@yahoo.com

4 UNAN-Managua/FAREM-Estelí. Correo electrónico: josuerod20@yahoo.com

5 UNAN-Managua/FAREM-Estelí. Correo electrónico: kenny.lb@hotmail.com 


\title{
Phenological behavior in three altitudinal ranges of species; Enterolobium cyclocarpum, Maclura tinctoria. Esteli, Nicaragua
}

\author{
Mariela Lisbeth Hernández Olivas ${ }^{1}$ \\ Beneyda Leticia Montalván Calderón ${ }^{2}$ \\ Ana María Flores Briones ${ }^{3}$ \\ Josué Tomas Urrutia Rodríguez ${ }^{4}$ \\ Kenny López Benavides 5
}

\section{RESUMEN}

Deterioration presented by tropical forest areas of Nicaragua, as a result of human actions, has resulted in the fragmentation and reduction of forests. Currently phenological studies have taken great importance, emphasizing mainly on biological processes of survival and reproductive success of the species, in order to improve the future distribution and adaptation to climate change; since variations on phenological stages have been observed.

The study consisted of evaluating the phenological behavior in three altitudinal ranges of tree species Enterolobium cyclocarpum, Maclura tinctoria in the dry tropical forest of northern Esteli Nicaragua, as to obtain information on the phenology of these species to contribute to the recovery and conservation of such. Phenological behavior was evaluated in three altitude ranges: lower, middle and upper. The observation of phenological characteristics was based on the methodology of Fournier (1974). The data obtained were analyzed using non-parametric test of Kruskal-Wallis variance. Individuals of the species M. tinctoria showed significant difference in the percentage of leaves, sprouts and fruits in the altitudinal range of 700-800 m; and E. cyclocarpum were only in leaves and sprouts in the altitudinal range of 900-1000 m. The specie Enterolobium cyclocarpum is the best suited at different heights, behaving consistently, and it is recommended for use in reforestation programs.

Keywords: Phenology, altitudinal ranges, Maclura tinctoria, Enterolobium cyclocarpum

1 UNAN-Managua/FAREM-Estelí. E-mail: marielalisbethhernandez@yahoo.es

2 UNAN-Managua/FAREM-Estelí. E-mail: mbeneydaleticia@yahoo.com

3 UNAN-Managua/FAREM-Estelí. E-mail: afloresbriones@yahoo.com

4 UNAN-Managua/FAREM-Estelí. E-mail: josuerod20@yahoo.com

5 UNAN-Managua/FAREM-Estelí. E-mail: kenny.lb@hotmail.com 


\section{INTRODUCCIÓN}

El bosque seco tropical es uno de los ecosistemas que se ha visto más afectado convirtiéndose en uno de los más amenazados por la sobreexplotación que se ha venido dando en plantaciones naturales en la mayoría de regiones del planeta y especialmente en Nicaragua, estimándose que queda menos del $1,5 \%$ de lo que originalmente existía (Betancourt, A. 1987).

El estudio tiene como propósito evaluar el comportamiento fenológico de especies propias del Bosque Tropical Seco (E. cyclocarpum $J$ y M. tinctoria $L)$ a tres rangos altitudinales. Esto nos permitirá comprobar si individuos de una misma especie al encontrarse a tres alturas diferentes presentan variaciones en sus etapas fenológicas de: floración, fructificación, hojas y rebrotes. Además se tendrá información sobre la fenología de estas especies arbóreas, contribuyendo de esta manera a su conservación.

El conocimiento y la comprensión de los patrones fenológicos de especies arbóreas en ecosistemas naturales es de interés básico no solo en estudios de biodiversidad, productividad y organización de las comunidades y de las interacciones de las plantas con la fauna, sino también en programas de conservación de recursos genéticos, manejo forestal y viveros forestales (Huxley, 1983).

Actualmente los estudios fenológicos han tomado una mayor importancia debido al proceso de calentamiento global (Chuine y Beaubien, 2001). Es por ello que se vuelve importante la iniciativa de su conservación llevando a cabo programas de investigación y reproducción de las mismas, ya que en la actualidad estos estudios son escasos para la región del trópico seco y poco se sabe de la fenología de las especies de árboles en la región, a pesar de la importancia que representan dichas especies en los ecosistemas.

\section{MATERIALES Y MÉTODOS}

\section{Ubicación del área de estudio}

El estudio se llevó a cabo en el Bosque Tropical Seco del Municipio de Estelí, abarcando los sectores de: a) La Campana y El Coyolito, que pertenecen al Paisaje Terrestre Protegido Miraflor-Moropotente, con alturas que oscilas entre 500 a $1200 \mathrm{msnm}$, temperaturas entre 20 y $24{ }^{\circ} \mathrm{C}$, precipitaciones de 800 a $1200 \mathrm{~mm} /$ año, pendientes entre 4\% y $30 \%$, vegetación boscosa caducifolia o subperennifolio, de bosque primario intervenido y bosque secundario. b) La comunidad el Limón perteneciente a la zona de amortiguamiento de la Reserva Natural Tisey - Estanzuela, con alturas que oscilan entre 800 a 910 msnm y temperaturas promedio de 24 a $26^{\circ} \mathrm{C}$, la topografía es ondulada, los suelos de textura franco arcillosa, sus bosques son de galería (Zeledón, 2002).

Se evaluó el comportamiento fenológico de las especies (E. cyclocarpum Jy M. tinctoria L) in situ a diferentes rangos altitudinales: zona baja, 700 a $800 \mathrm{msnm}$ (El Limón), zona media, 900 a 1000 msnm (El Coyolito), y zona alta de 1,100 a 1,200 msnm (La Campana).

Para la observación fenológica se seleccionaron por orden de aparición en el bosque (Fournier \& Charpantier 1975). 10 árboles por cada una de las especies con características morfológicas similares (Jiménez et al, 1996), y por rango altitudinal. las etapas fenológicas observadas fueron : porcentajes de hojas, rebrotes, flores, y frutos, basándose en la metodología de Fournier (1974), que consiste en la aplicación de la siguiente escala: de 0 a 4; donde 0 es ausencia de la etapa fenológica, 1 corresponde de 1 a $25 \%, 2$ : de 26 a 50\%, 3: de 51 a $75 \%$ y 4 : de 76 a $100 \%$ de presencia de la característica, la cual facilito la evaluación cuantitativa de las diferentes características fenológicas de cada individuo. Las observaciones se realizaron quincenalmente, durante un periodo de ocho meses (Abril 2012 a Octubre 2012). 
Para la observación de las características fenológicas se dividió la copa del árbol en cuatro cuadrantes, tomando los datos por separados, para sumar y promediar al final el porcentaje de: Flores, frutos, hojas y rebrotes producidos por cada árbol seleccionado. Para realizar esta actividad fue necesario el trabajo de un observador, con el objetivo de que los datos no varíaran por influencia de varios observadores, haciendo uso de los binoculares.

\section{Análisis estadístico}

Se determinó la normalidad de los datos, utilizando la prueba estadística de Kolmogorov - smirnov (KS) para muestras independientes. Posteriormente se realizaron pruebas de normalización de datos, a través de logaritmos naturales, raíz cuadrada y arco seno de la raíz cuadrada. Después se aplicaron Análisis de Varianza no Paramétricos de Kruskall Wallis.

Para el análisis de datos se utilizaron los software: Excel versión 2013, SPSS versión 20 e InfoStat versión 2013.

\section{RESULTADOS Y DISCUSIÓN}

Comportamiento fenológico en su ambiente natural de la especie arbórea Maclura tinctoria en tres rangos altitudinales

\section{Porcentaje de hojas y rebrotes}

Se encontraron diferencias significativas $(\mathrm{P}<0.0282)$, en el porcentaje de hojas de los individuos de Maclura tinctoria del rango altitudinal de 700 a $800 \mathrm{msnm}$ con respecto al rango de 1100 a $1200 \mathrm{msnm}$ y el rango de 900 a 1000 msnm que presenta características similares a los mencionadas anteriormente (Ver figura, A). De igual forma ocurrió en el porcentaje de rebrotes presentando diferencias significativas, siendo el valor de significación $\mathrm{P}<0.0015$. (Ver figura, $\mathrm{B}$ ).
A)
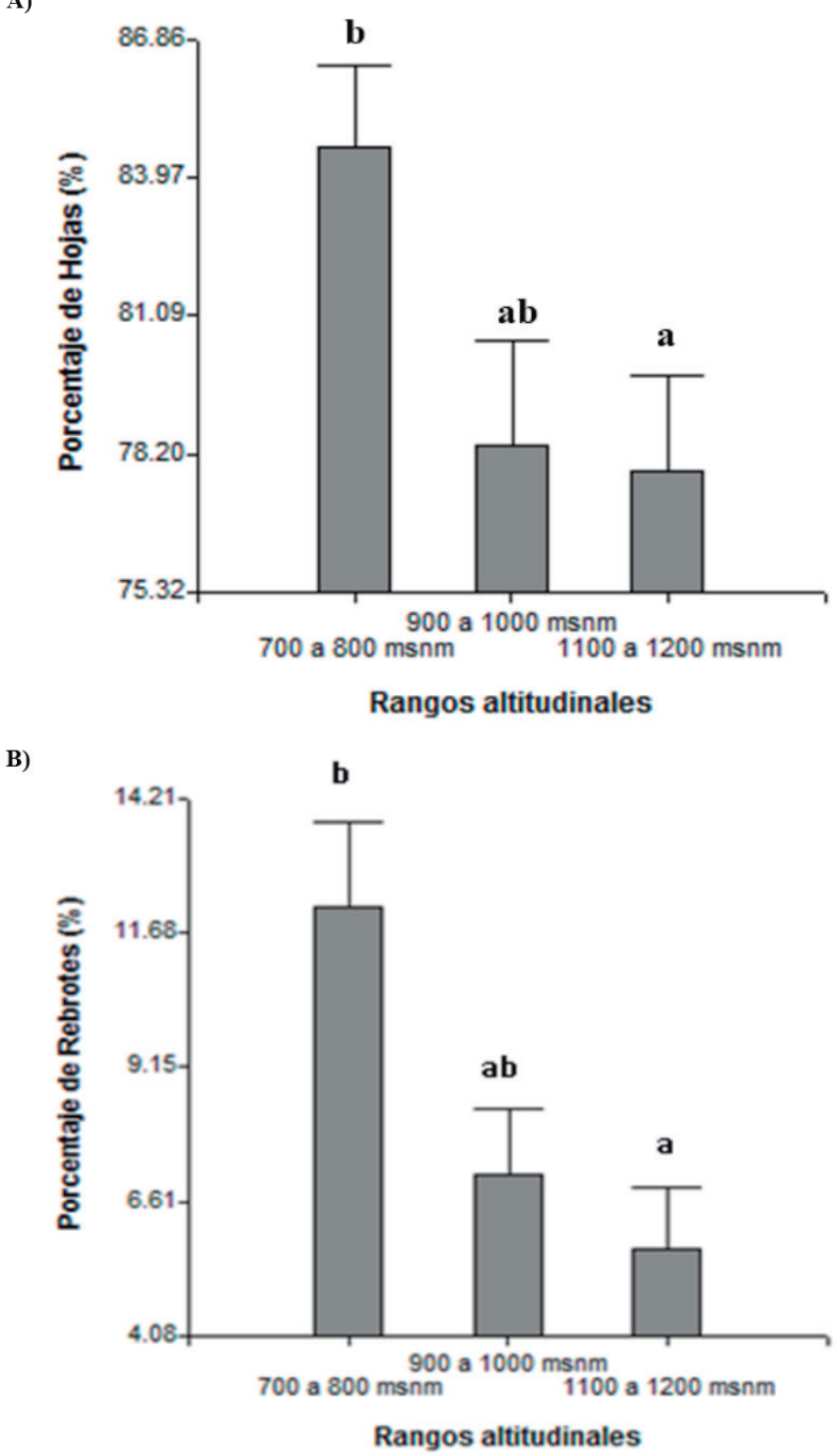

Figura 1. Características fenológicas de la especie Maclura tinctoria: porcentaje de hojas (A) y rebrotes (B), a tres rangos altitudinales.

Maclura tinctoria es una especie semi-decidua es decir que deja caer un alto porcentaje de sus hojas durante la temporada seca lo cual coincide con la producción de flores, convirtiéndose así en un indicador del inicio de esta fenofase. Según Scott (1966) una gran mayoría de los árboles que habitan estas regiones con períodos de sequía prolongados están, en una u otra forma, adaptados a estas condiciones ambientales. 
Es preciso destacar que la aparición de rebrotes y presencia de hojas durante los meses estudiados (abril a octubre) permaneció de manera irregular entre los individuos por rango altitudinal; ya que el brote de hojas inicia de manera casi simultánea con la caída, por lo que no se alcanzan a observar durante mucho tiempo al individuo sin follaje y la mayor producción de hojas nuevas se registra durante la temporada de lluvias, esto es, una vez que finaliza la cosecha de los frutos y continúa durante buena parte del año.

Sin embargo autores como Borchet (1994), asignan el papel más importante en el control de la duración de la fase de aparición de rebrotes y presencia de hojas a la interacción entre la edad de estas hojas y el inicio de la estación seca.

Scott (1966) reporta que la fenología de los árboles tropicales puede responder también a señales ambientales. Sin embargo autores como Reich y Borchert (1984), señalan que algunos de estos comportamientos son facultativos debido a que individuos de la misma especie que crecen en sitios diferentes o en el mismo sitio pueden desplegar marcadas diferencias fenológicas entre sí.

\section{Porcentaje de flores y frutos}

No se encontraron diferencias significativas $(\mathrm{P}>0.3260$, $\mathrm{ns}$ ), en el porcentaje de flores; los individuos de la especie Maclura tinctoria encontrados en los tres rangos altitudinales de 700 a $800 \mathrm{msnm}, 900$ a $1000 \mathrm{msnm}$ y 1100 a $1200 \mathrm{msnm}$, no presentaron variaciones en la característica fenológica (Ver figura, C).

Sin embargo en la característica de fructificación si mostraron diferencia significativa $(\mathrm{P}<0.0497)$; indicando que los individuos del rango altitudinal de 700 a $800 \mathrm{msnm}$ presentan un mayor porcentaje de frutos, con respecto a los demás rangos (Ver figura, D).
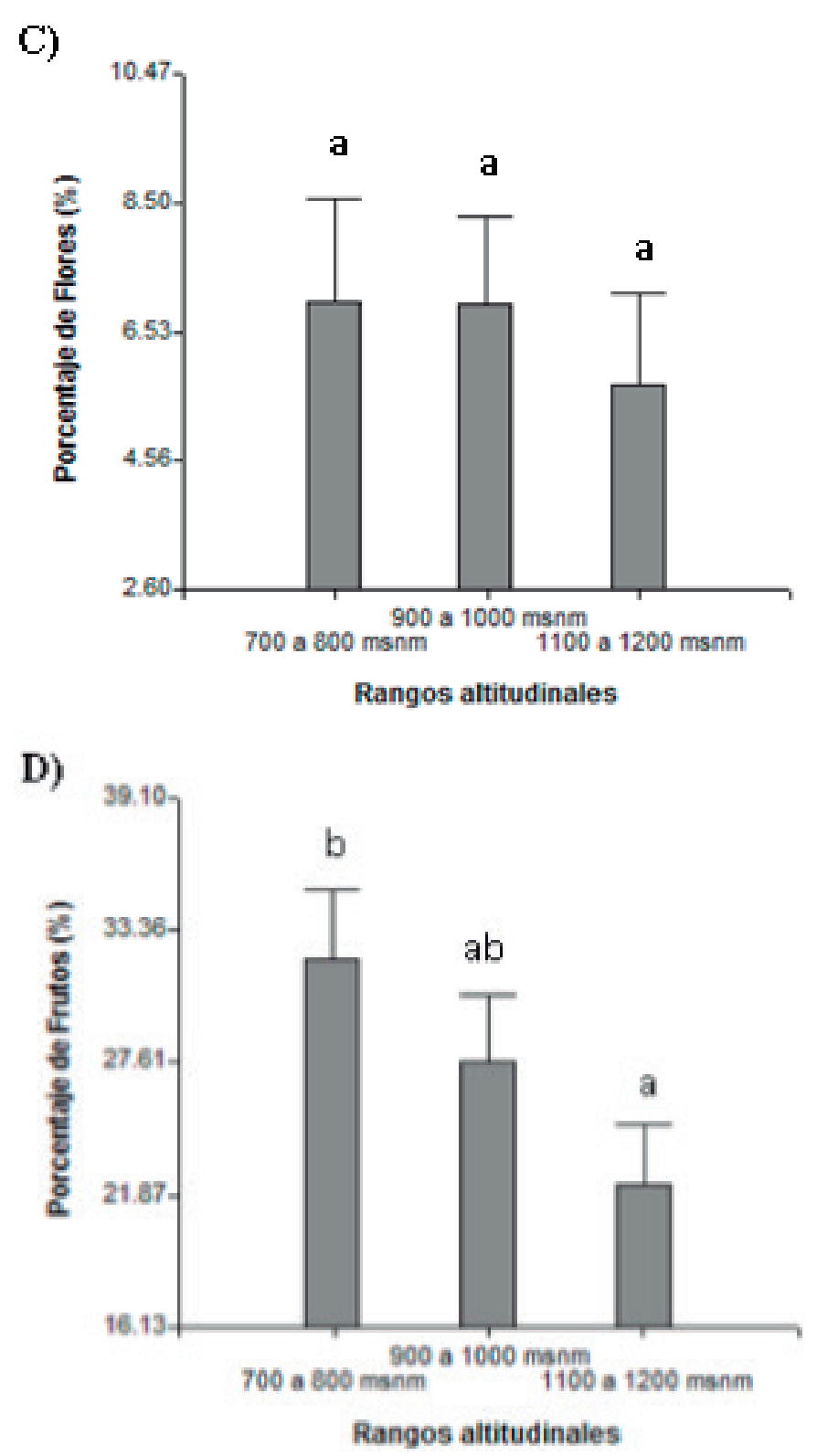

Figura 2. Características fenológicas de la especie Maclura tinctoria: porcentaje de flores (C) y frutos (D) en tres rangos altitudinales.

Los resultados obtenidos reflejan que los individuos de Maclura tinctoria a diferentes rangos altitudinales muestran comportamiento fenológico diferentes; observándose que la parte baja es la que presenta mayores porcentajes en las etapas de fructificación, hojas y rebrotes con respecto a la zona media y alta.

A Scott (1966) le parece un tanto paradójico que, la mayor floración se encuentre concentrada en la época 
seca, que lógicamente debe ser la más crítica para las plantas. Esta observación de Scott es muy atinada; sin embargo, hay algunos hechos que parecen sugerir que la concentración de floración en esta época es más bien el resultado de un largo proceso de adaptación, parte de la misma dinámica de estas formaciones. Estos resultados están relacionados al éxito reproductivo y es posible que esta especie pueda producir más frutos en las zonas bajas que en las altas, porque los individuos que se encuentran ahí responden mejor a las condiciones bióticas y abióticas del ambiente.

Según lo que expresa Gómez (2010), el análisis de los períodos de ocurrencia de las etapas fenológicas de las especies en las zonas bajas exhibe una mayor estacionalidad que las especies de las zonas altas esto corrobora lo observado en el estudio; esto no quiere decir que se esté produciendo una mejor adaptación a menores alturas, ya que la especie es propia de un hábitat de alturas a nivel del mar y los 1,200 msnm (Zamora, N.1999).

Algunos estudios realizados de fenología de las especies, encontraron que de manera general, la identificación de los patrones de fructificación se torna difícil en algunas especies, ya sea porque los frutos de la misma cosecha permanecen en el árbol por largo tiempo; o por el contrario, desaparecen rápidamente de la copa del árbol porque son muy apetecibles para la fauna silvestre, o porque son abortados, por lo que existe variación en la producción de frutos en los diferentes individuos de la misma especie, y que ésta depende de la intensidad y la duración de la estación seca y de las formas de vida (Foster, 1990).

De igual forma se considera lo expresado por Mejía (1990), quien indicó que la altitud, influye en la fenología de las plantas, nuestros resultados confirman lo expresado por Mejía, porque se corroboró que los individuos de la especie Maclura tinctoria al encontrarse en diferentes altitudes presentan variación en sus etapas fenológicas. Por su lado Borchert (1996), analizó las diferencias fenológicas de 18 especies tropicales mediante el uso de colecciones de herbario, y encontró que el ámbito de la variación geográfica tiene un rol importante en el comportamiento de las especies y destacó que las diferencias en la duración y la intensidad de la época seca afectan mucho la sincronía de la floración, y por ende la de la fructificación

Comportamiento fenológico de la especie arbórea Enterolobium cyclocarpum Jacq en tres rangos altitudinales

\section{Porcentaje de hojas y rebrotes}

Existe diferencia significativa en el porcentaje de hojas en los individuos de Enterolobium cyclocarpum, ya que el valor de significación es de $\mathrm{P}<0.0011$; el rango de 900 a $1000 \mathrm{msnm}$ presenta el mayor porcentaje de la característica respecto al rango de 700 a $800 \mathrm{msnm}$ que presentó los valores más bajos; en cambio el rango de 1100 a $1200 \mathrm{msnm}$ presenta características similares de los demás rangos (Ver figura, E). En la etapa de rebrotes también se presentó diferencia significativa $(\mathrm{P}<0.0002)$; los rangos de 900 a 1000 msnm y 1100 a $1200 \mathrm{msnm}$ presentan los mejores porcentajes de rebrotes, respecto al de 700 a 800 msnm que alcanzó los valores más bajos (Ver figura, F).

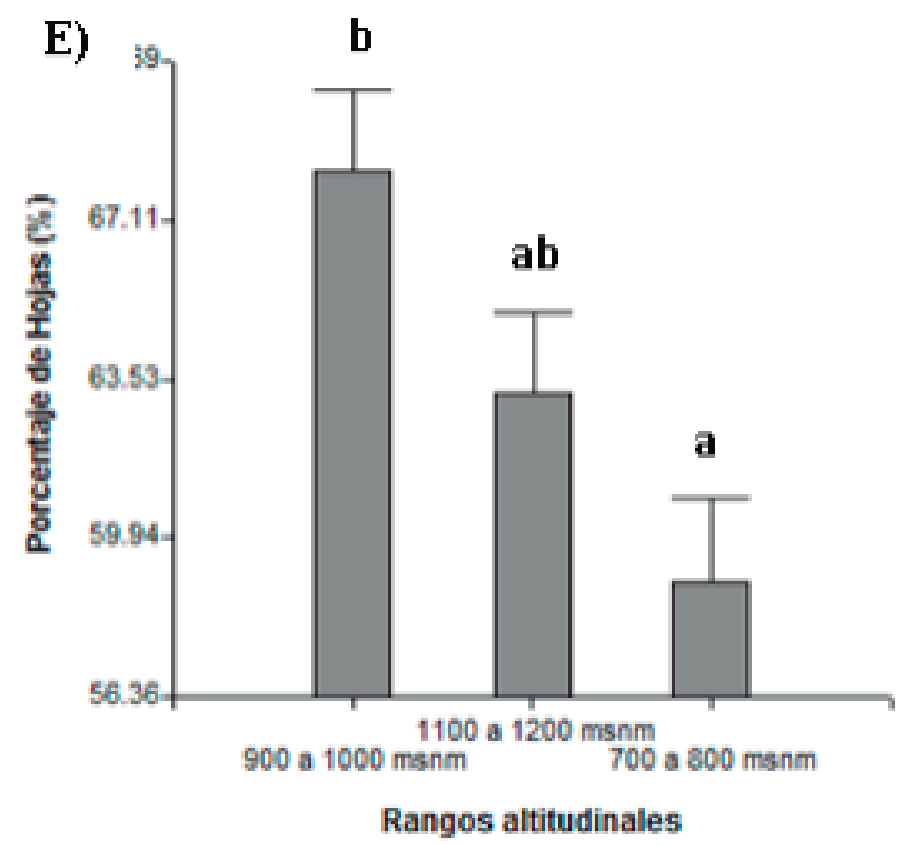




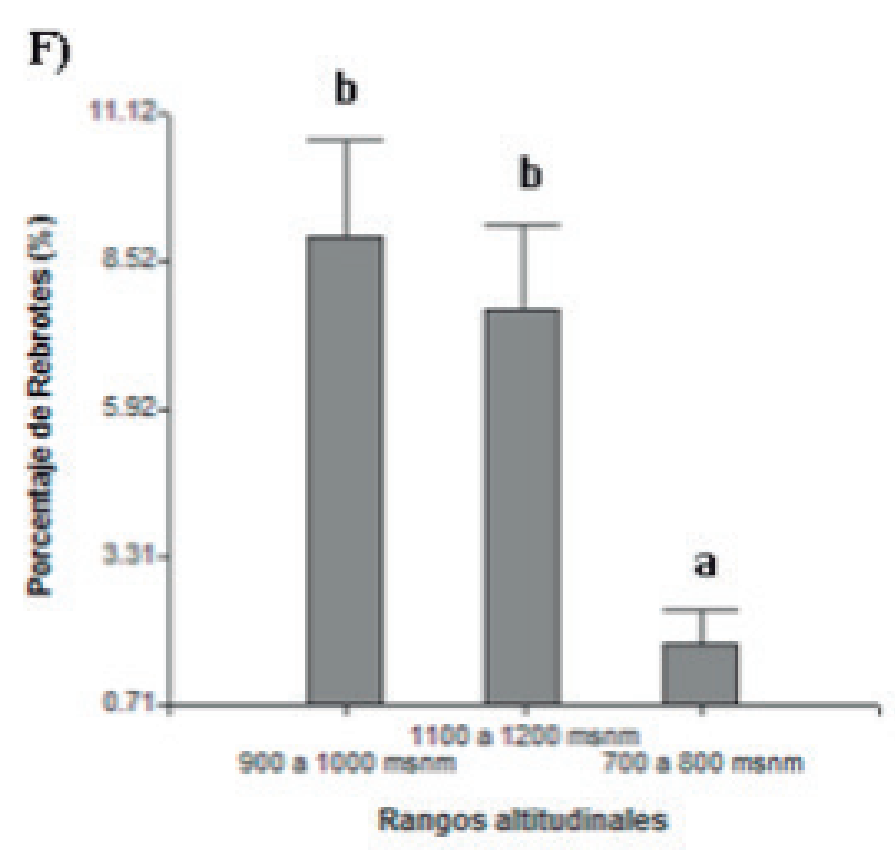

Figura 3. Características fenológicas de la especie Enterolobium cyclocarpum: porcentaje de hojas $(\mathrm{E})$ y rebrotes $(\mathrm{F})$ en tres rangos altitudinales.

El Guanacaste coloniza una gran variedad de hábitat, a pesar de que es común a elevaciones bajas se le puede encontrar creciendo de manera natural hasta los 900 msnm y se le ha plantado a elevaciones de $1100 \mathrm{msnm}$. Es un árbol semi-caducifolio, es decir que no pierde las hojas en su totalidad. Su defoliación está altamente asociada con la floración y la formación de los frutos, eventos que a su vez se presentan en los meses más secos del año.

Es una especie que presenta patrón fenológico de copa, por lo que tiene la sorprendente capacidad de producir y expandir el follaje antes de la caída de las primeras lluvias, denominándose especie de brotación temprana (Frankie et al. 1974), comportamiento ya expresado por Richards (1996), quienes comentan que una gran proporción de árboles de bosques tropicales nunca están desnudos de hojas y que la caída de hojas viejas es rápidamente seguida por la aparición de hojas jóvenes. Caso que sucede en la especie Enterolobium cyclocarpum que la caída de hojas viejas es acompañada por hojas nuevas o rebrotes.
La brotación de las hojas en esta especie es acompañada por la apertura de las flores y la formación temprana de frutos, los cuales luego interrumpen su desarrollo durante la estación lluviosa (Rojas et al. 2007). Existen algunas hipótesis que señalan los cambios en el contenido de humedad del suelo (Reich \& Borchert 1984), el fotoperiodo (Bullock 1990), la temperatura (Gómez \& Fournier 1996) y ritmos endógenos (Fournier \& Fournier 1986), como posibles activadores de la brotación temprana y a las reservas internas de agua como las fuentes que soportan la brotación del follaje (Borchert 1994b).

Autores como Orozco (1992) señalan que muchas especies pueden tener comportamientos distintos en diferentes sitios, caso que se presentó en las etapas de hojas y rebrotes presentando diferencia significativa principalmente en el rango altitudinal de 900 a 1000 msnm, respecto a los demás rangos.

\section{Porcentaje de flores y frutos}

No existen diferencias significativas en el porcentaje de flores y frutos, los individuos de la especie Enterolobium cyclocarpum, ya que el valor de significación es $(\mathrm{P}>0.05)$, no se presentó variación alguna en la característica en ninguno de los rangos (Ver figura, G, H).

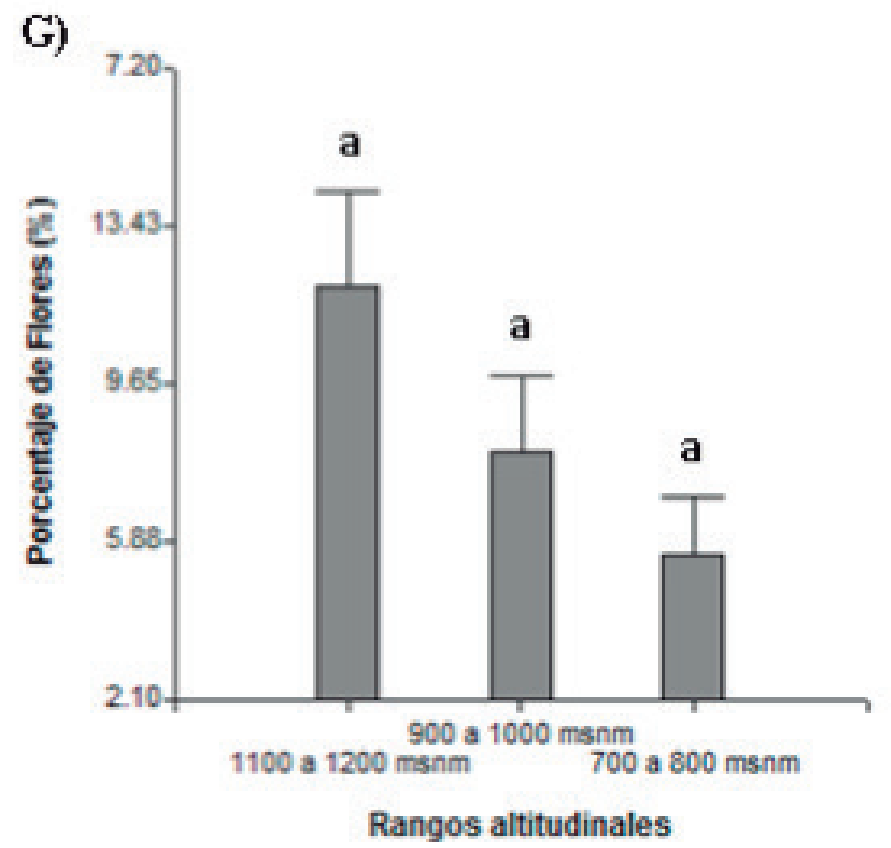




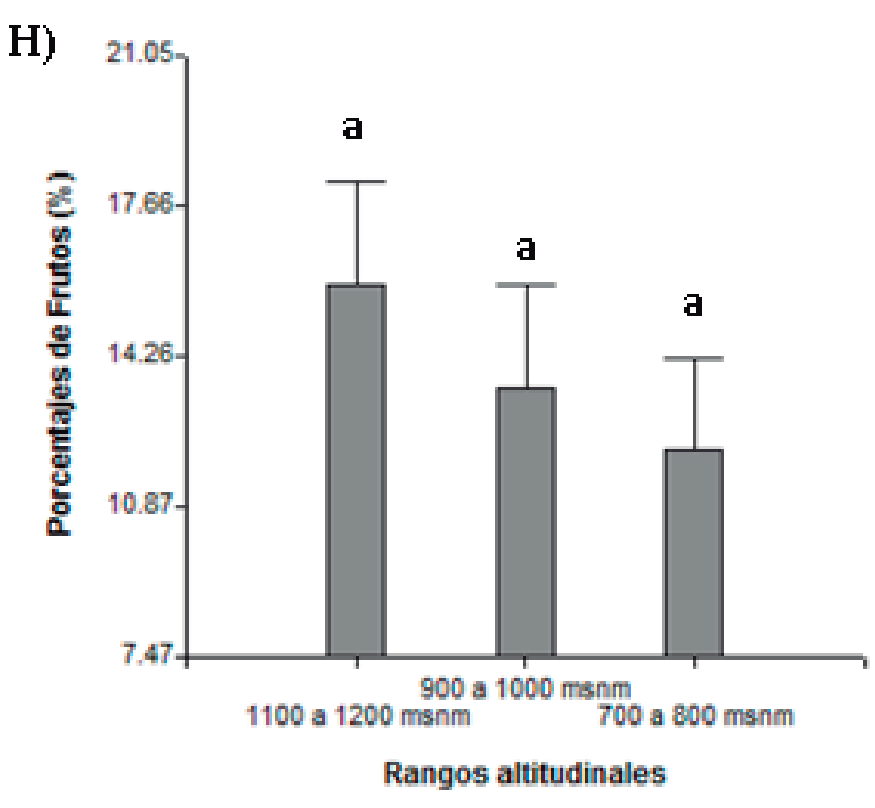

Figura 4: Características fenológicas de la especie arbórea Enterolobium cyclocarpum: porcentaje de flores $(\mathrm{G})$ y frutos

$(\mathrm{H})$, en tres rangos altitudinales.

Según Blaser y Camacho (1991), la ausencia de diferencias significativas entre sitios en la mayoría de las especies demuestra que estas tienen comportamientos fenológicos similares en un área determinada. Esto se puede relacionar con lo encontrado en las etapas de floración y fructificación de los individuos de la especie Enterolobium cyclocarpum que en los tres rangos altitudinales no presentaron diferencia significativa alguna.

Asimismo, Aide (1988) considera que las especies al desarrollar la floración y la fructificación pierden algo de su follaje, por lo tanto, la caída del follaje favorece la floración, caso contrario al que sucede con las especie Maclura tinctoria y Enterolobium cyclocarpum que mantiene su follaje al mismo tiempo que florecen y fructifican. Por otro Pires-O'Brien (1995), destaca que la falta de lluvia, podría el principal factor responsable de que los árboles florezcan en períodos secos, evitando la pérdida masiva de flores.

La floración y fructificación son características relevantes, que determina el rendimiento reproductivo de las especies, ya que puede poner restricciones en el uso de los recursos estacionales, tales como luz, agua y polinizadores por parte de las plantas (Marco et al., 2000). La mayoría de las especies de los bosques tropicales presentan variación estacional en la aparición de nuevas hojas, flores y frutos; este patrón sugiere que los cambios fenológicos representan adaptaciones a factores bióticos y/o abióticos, siendo el clima el factor principal (Van Schaik et al., 1993).

Borchert (1980) descarta los factores climáticos como determinantes primordiales de la floración de las especies arbóreas en los trópicos y considera que los patrones fenológicos son determinados, principalmente, por procesos periódicos endógenos $\mathrm{y}$ en forma secundaria como adaptación a cambios ambientales.

Las diferencias encontradas en el comportamiento fenológico y en la productividad de la especie respecto a otros estudios (Vélez, 1992) puede deberse a metodologías diversas, ya que la duración de tales estudios (menos de 3 años) pudo no permitir la clara identificación del ciclo reproductivo. De acuerdo con Newstron et al. (1994), como muchas especies presentan ciclos fenológicos multianuales, se requieren períodos de observación prolongados, por lo menos 5 años, para describir completamente estos patrones.

\section{CONCLUSIÓN}

Los individuos de la especie Maclura tinctoria mostraron diferencias significativas en el porcentaje de hojas, rebrotes y frutos; en la zona baja (700 a $800 \mathrm{msnm}$, respecto a las demás zonas; a diferencia de las flores. En cambio en Enterolobium cyclocarpum mostraron diferencia significativa los individuos de la zona media (900 a 1000) en el porcentaje de hojas y rebrotes; las etapas de floración y fructificación, no mostraron diferencia significativa alguna. 


\section{BIBLIOGRAFÍA}

Aide, T.M. (1988). Herbivory as a selective agent on the timing of leaf production in a tropical understory community. Nature. Vol. 336; p. 574-575.

Betancourt, A. (1987). Silvicultura especial de árboles maderables tropicales. Cuba. Científico Técnica. p. 342-356.

Borchert, R (1980). Phenology and ecophysiology of tropical trees: erythrina poeppigiana O.F. Cook. Ecology 61: 1065- 1074.

Borchert, R. (1994) b. Soil and stem water storage determine phenology and distribution of tropical dry forest trees. Ecology 75(5):1437-1449.

Borchert R(1996). Phenology and flowering periodicity of Neotropical dry forest species: evidence from herbarium collections. J. Trop. Ecol. Vol. 12; p. 65-80.

Blaser, J. \& M. Camacho. (1991). Estructura, composición y aspectos silviculturales de un bosque de roble (Quercus spp.) del piso montano en Costa Rica. CATIE, Turrialba, Costa Rica. 68p.

Bullock, S; Solís-Magallanes, A. (1990). Phenology of canopy trees of a tropical deciduos forest in México. Biotrópica 22(1):22-35.

Chuine, I., and E. Beaubien. (2001). Phenology is a major determinant of temperate tree range. Ecology Letters 4:500-510.

Frankie, G; Beker, H; Opler, P.1974. Comparative phenological studies of trees in tropical wet and dry forests in the lowlands of Costa Rica. Journal of Ecology. 62:881-919 Forest Service. 1943. The forests of Costa Rica. Washington, DC: U.S. Department of Agriculture. 48 p.

Foster R. (1990). Long-term change in the successional forest community of the Rio Manu Floodplain. In: A.H. Gentry ed. Four Neotropical rainforests. Yale Univ. Press, New Haven, CT.: 565-572.

Fournier, L. y C. Charpantier. (1975). El tamaño de la muestra y la frecuencia de las observaciones en el estudio de las características fenológicas de los árboles tropicales. Turrialba 25: 45-48.
Fournier, L; Fournier, M. (1986). Fenología y ecofisiología de Gliricidia sepium "Madero Negro” en Ciudad Colón, Costa Rica. Rev. Biol. Trop. 34(2):283-288.

Gómez,P; Fournier, L.(1996). Fenología y ecofisiología de dos poblaciones de Tabebuia rosea ("Roble de Sabana") en Costa Rica (Bignoniaceae). Rev. Biol. Trop. 44(1):61-70.

Gómez R. Martha Ligia(2010). Fenología reproductiva de especies forestales nativas presentes en la jurisdicción de CORANTIOQUIA, un paso hacia su conservación. Volumen I /, Corporación Autónoma Regional del Centro de Antioquia, CORANTIOQUIA. Medellín: CORANTIOQUIA, 228 p.ISBN: 978-958-99363-3-7.

Marco, D.E., A.A. Calviño and Sergio Páez. (2000). Patterns of Flowering and Fruiting in Papulations of Larrea divaricata in Dry Chaco (Argentina). Journal of Arid Environments 44:327-346.

Mejía, M.G. (1990). Fenología: Fundamentos y métodos. En. Seminario Taller en Semillas Forestales Tropicales. ( $2^{\circ}$ Bogotá, Colombia). Memoria. Ed. T. Triviño. Bogotá, CO. p. 65-79.

Newstrom, L.E., Frankie, G.W. y Baker, H.G. (1994). A new classification for plant phenology based on flowering patterns in lowland tropical rain forest trees at La Selva, Costa Rica. Biotropica, 26(2): 141-159.

Orozco, L. (1992). Estudio ecológico y de estructura horizontal de seis comunidades boscosas de la Cordillera de Talamanca, Costa Rica. CATIE, Turrialba, Costa Rica. 33 p.

Pires-O'Brien, M.J.; O’Brien, C.M. (1995). Aspectos evolutivos da fenología reprodutivadas árvores tropicais. Belém: FCAP, 25 p.

Preuhsler, T., A. Bastrup-Birk \& E. Beuker. (2006). Manual on methods and criteria for harmonized sampling, assessment, monitoring and analysis of the effects of air pollution on forests Part IX Phenological Observations. United Nations Economic Commission for Europe Convention on Long-Range Transboundary Air Pollution. 
International Cooperative Programme on Assessment and Monitoring of Air Pollution Effects on Forests. Consultada el 22 de agosto del 2006, (http://www.metla.fi/eu/icp/phenology/ manual).

Reich, P.B, Borchert, R (1984). Water stress and tree phenology in a tropical dry forest in the lowlands of Costa Rica. Journal of Ecology 72: 61-74.

Richards, P. W. (1996). The tropical rain forest. Cambridge University Press. Cambridge, U.K. 575 pp.

Rojas-Jiménez, K; Hoolbrok, NM; Gutiérrez, M. (2007). Dry-season leaf flushing of Enterolobium cyclocarpum (ear-pod tree): above and belowground phenology and water relations. Tree Physiology 27:1561-1568.

Scott, N. (1966). Ecologically importa 111 aspects 01 the climates of Costa Rica, Organization for Tropical
Studies, Costa Rica, 26 pp. (mimeografiado).

Van Schaik, C; Terborgh, J. W. y Wright, S. J. (1993). The phenology of tropical forests: adaptative significance and consequence for primary consumers.Annu. Rev. Ecol. Syst. 24: 353-377.

Vélez, G. (1992). Estudio fenológico de diecinueve frutales silvestres utilizados por las comunidades indígenas de la región de Araracuara - Amazonía colombiana. Colombia Amazónica, 6: 135-186.

Zamora, N.; González J. \& Poveda, L. J. en prep. (1999). Árboles y Arbustos del Bosque Seco de Costa Rica. Instituto Nacional de Biodiversidad, Costa Rica.

Zeledón Alba Marina (2002). Estructura del bosque tropical seco y sus usos, en las comunidades de El Limón, el Dorado y el Coyolito - Estelí, Estelí marzo 2004. Universidad Autónoma de Barcelona (UAB - FAREM). 